\title{
Reverse triggering with breath stacking during mechanical ventilation results in large tidal volumes and transpulmonary pressure swings
}

\author{
Henry K. Su ${ }^{1,2^{*}}$, Stephen H. Loring ${ }^{2}$, Daniel Talmor ${ }^{2}$ and Elias Baedorf Kassis ${ }^{3}$
}

(C) 2019 Springer-Verlag GmbH Germany, part of Springer Nature

\section{Dear Editor,}

Reverse triggering with breath stacking is a form of dyssynchrony in mechanically ventilated patients with ARDS which may contribute to ventilator-induced lung injury (VILI) through the delivery of large tidal volumes (Vt) [1]. It occurs when an initially passive breath is followed during the expiratory phase by a reflexive or purposeful diaphragmatic contraction of sufficient force to trigger the delivery of an additional breath. Differentiating reverse triggering from other causes of breath stacking (e.g. double triggering) is challenging using standard time tracings. Our group has recently employed the Campbell diagram, which uses esophageal pressure $\left(P_{\mathrm{es}}\right)$ to demonstrate the pressure-volume characteristics of the chest wall, to phenotype breathing patterns [2]. This is an ideal tool for identifying the subtle inspiratory differences associated with reverse triggering.

We generated Campbell diagrams in patients with ARDS from a prior study [3] to identify reverse trigger events by visual inspection (Fig. 1). Approval was obtained from the Institutional Review Board at Beth Israel Deaconess Medical Center. Subjects received lung protective ventilation (control mode with Vt $6 \mathrm{cc} / \mathrm{kg}$ ) with PEEP adjusted per protocol and sedation adjusted on an individual basis. Maximal transpulmonary pressure (PL) fluctuations and volume during reverse trigger events were recorded for each patient and compared to the closest prior normal breath. For patients exhibiting

\footnotetext{
*Correspondence: henry_su@hms.harvard.edu

${ }^{1}$ Harvard Medical School, 25 Shattuck Street, Boston, MA 02215, USA

Full author information is available at the end of the article
}

multiple reverse triggers, the average values for the first, last and middle (defined as the $n / 2+1$ event, rounded down, where $\mathrm{n}$ is the total number of events) events were used. Statistical analysis was performed with a paired, two-tailed $t$ test.

Of 56 total patients, 17 exhibited reverse triggering events. Reverse triggering was found in 171 of 3849 total breaths over 144.7 min of recording. Diaphragmatic contraction was initiated during the late-inspiratory phase and early-expiratory phase in $70(40.9 \%)$ and 101 (59.1\%) instances, respectively. Reverse triggers resulted in significantly larger Vt $(593.2 \pm 157.8 \mathrm{~mL}$ vs. $413.8 \pm 84.7 \mathrm{~mL}$, $p=0.0002)$ and maximal PL fluctuations $(22.6 \pm 7.1 \mathrm{~cm}$ $\mathrm{H}_{2} \mathrm{O}$ vs. $17.7 \pm 5.9 \mathrm{~cm} \mathrm{H}_{2} \mathrm{O}, p=0.001$ ) compared to passive breaths.

The clinical consequences of reverse triggering may depend on the context, frequency and degree of excess $\mathrm{Vt}$ and PL fluctuation. In a milieu of other dyssynchronous patient efforts, reverse triggering may be harmful and contribute to VILI [1], and our work suggests the mechanism is not just volutrauma but also barotrauma, which is an important distinction. On the other hand, it is possible that infrequent/rare events could have the opposite impact in other patients, in whom we observed reverse triggering intermittently during periods of relative synchrony. Just as "sighs" on the ventilator have been postulated to help maintain lung recruitment and improve oxygenation [4], occasional reverse triggering could similarly act as miniature recruitment maneuver. Indeed, diaphragmatic contraction during the expiratory phase might help maintain lung recruitment, even when the effort is insufficient to trigger an additional

\section{Springer}



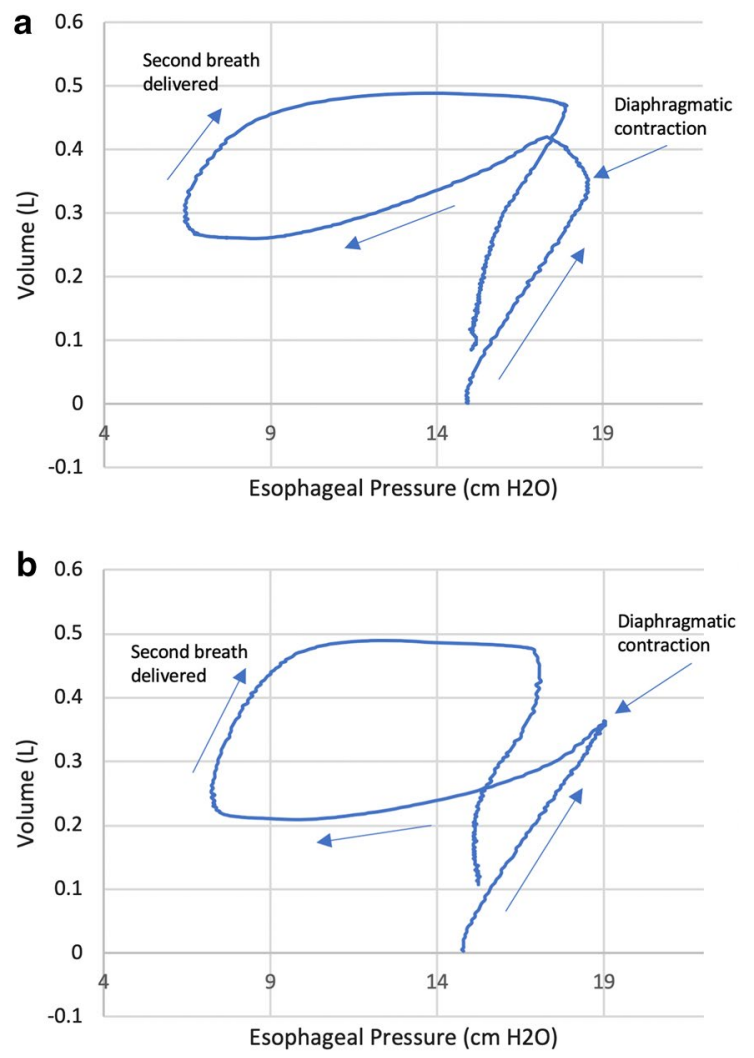

Fig. 1 Campbell diagrams of reverse trigger events. a Reverse trigger and stacked breath following a diaphragmatic contraction initiated during the late-inspiratory phase of an initial ventilator-initiated, passive breath. Note that the negative $P_{\text {es }}$ deflection, which represents diaphragmatic contraction, starts prior to completion of the inspiratory phase, when the full volume of the initial breath has not yet been completely delivered. b Reverse trigger and stacked breath following a diaphragmatic contraction initiated during the earlyexpiratory phase of an initial ventilator-initiated, passive breath. Note that the negative $P_{\text {es }}$ deflection starts as the expiratory phase begins, after the intended volume has already been delivered

ventilator-delivered breath [5]. Differentiating the contexts in which these events are potentially harmful or helpful is critical. To further study these phenomena, we are currently working towards using the Campbell diagram in a more comprehensive analysis on a larger data set.

\section{Electronic supplementary material}

The online version of this article (https://doi.org/10.1007/s00134-019-05608-y) contains supplementary material, which is available to authorized users.

\section{Author details}

${ }^{1}$ Harvard Medical School, 25 Shattuck Street, Boston, MA 02215, USA.

${ }^{2}$ Department of Anesthesia, Critical Care and Pain Medicine, Beth Israel Deaconess Medical Center and Harvard Medical School, 330 Brookline Ave, Boston, MA 02215, USA. ${ }^{3}$ Division of Pulmonary, Critical Care and Sleep Medicine, Beth Israel Deaconess Medical Center and Harvard Medical School, 330 Brookline Ave, Boston, MA 02215, USA.

\section{Compliance with ethical standards}

\section{Conflicts of interest}

On behalf of all authors, the corresponding author states that there is no conflict of interest.

\section{Publisher's Note}

Springer Nature remains neutral with regard to jurisdictional claims in published maps and institutional affiliations.

Accepted: 20 March 2019

Published online: 28 March 2019

\section{References}

1. de Haro C, López-Aguilar J, Magrans R et al (2018) Double cycling during mechanical ventilation: frequency, mechanisms, and physiologic implications. Crit Care Med 46(9):1385-1392

2. Baedorf Kassis E, Loring SH, Talmor D (2018) Lung volumes and transpulmonary pressure are decreased with expiratory effort and restored with passive breathing in ARDS: a reapplication of the traditional Campbell diagram. Intensive Care Med 44(4):534-536

3. Talmor D, Sarge T, Malhotra A et al (2008) Mechanical ventilation guided by esophageal pressure in acute lung injury. N Engl J Med 359(20):20952104. https://doi.org/10.1056/NEJMoa0708638

4. Pelosi P, Cadringher P, Bottino $\mathrm{N}$ et al (1999) Sigh in acute respiratory distress syndrome. Am J Respir Crit Care Med 159(3):872-880. https://doi. org/10.1164/ajrccm.159.3.9802090

5. Pellegrini M, Hedenstierna G, Roneus A, Segelsjö M, Larsson A, Perchiazzi $G$ (2017) The diaphragm acts as a brake during expiration to prevent lung collapse. Am J Respir Crit Care Med 195(12):1608-1616. https://doi. org/10.1164/rccm.201605-0992OC 